LANDAU-97-TMP-2

hep-th/9704148

\title{
Scaling limit of the six vertex model in the framework of free field representation
}

\author{
Michael Lashkevich \\ Landau Institute for Theoretical Physics, GSP-1, 117940 Moscow V-334, Russia
}

\begin{abstract}
The scaling limit of the spectrum, $S$ matrix, and of the form factors of the polarization operator in the six vertex model has been found. The result for the form factors is consistent with the form factors of the sine-Gordon model found recently by Lukyanov. We discuss the origin of the structure of the free field representation for the sine-Gordon model at the critical coupling from the point of view of the lattice model.
\end{abstract}

April 1997

\section{Introduction}

In the recent years a considerable progress has been made in investigation of integrable models of twodimensional quantum field theory. $S$ matrices ${ }^{1}$ and form factors ${ }^{2}$ have been found in the framework of the bootstrap approach. A free field representation ${ }^{3}$ for the form factors of the sine-Gordon and Thirring models was proposed. But these achievements are based on some guesses and we need some additional physical grounds to understand better the nature of these results. The main problem can be formulated in the following way. It is possible to construct sets of form factors, that describe some local operators on the basis of the axiomatic approach. Moreover, it is possible construct the whole space of local operators. But it turns out very difficult to understand which operator corresponds to a given set of the form factors, and vice versa: if we consider a given operator, then which set of axiomatically allowed form factors corresponds to it?

A approach to lattice models of statistical mechanics based on the free field representation of correlation functions and form factors has been developed by the Kyoto group. ${ }^{4,5,6}$ In contrast to the situation in the quantum field theory this approach has a firm physical basis. There is a simple rule that allows one to construct a bosonic representative to any local lattice object. So it one can easily construct a set of form factors corresponding to each local lattice insertion. On the other hand, the lattice models are known to be described by models of quantum field theory at large scale in the vicinity of the critical point. So, it seems promising to investigate these models in the vicinity of the critical point to extract some information on the respective quantum field models. Our main strategy is the following. We consider a lattice theory and find form factors of some local insertions in the scaling limit. Then from some additional information, such as scaling dimensions, conservation laws, symmetries, mutual locality etc. we associate a local operator in the field theory to the insertion. This allows us to find to relate form factors to particular operators. Here we do not fulfil this program sequentially, but make some steps in this direction.

In this letter we consider the six vertex model $^{7}$ in the antiferroelectric region in the vicinity of the critical point. In the critical region this model is described by the $S U(2)$ invariant Thirring model or, equivalently, by the sine-Gordon model at the critical coupling. ${ }^{8}$ We analyze the spectrum, the $S$ matrix, and the form factors of the polarization operator $\sigma^{z}$. Our result does not match that obtained by Jimbo and Miwa, ${ }^{6}$ but we shall see that it is consistent with the formula for form factors in the sine-Gordon model, guessed recently by Lukyanov. ${ }^{9}$ We show the crucial role of the zero mode operators to the behavior of the form factors in the scaling limit, and show how to pass to a 'conjugate' pair of the zero mode operators simplifies the limit. 


\section{Spectrum and $S$ Matrix}

The six vertex model in the antiferroelectric region is described by two parameters $x$ and $\zeta$,

$$
0<x<1, \quad 1<\zeta<x^{-2},
$$

so that the weight matrix of the model is given by the trigonometric $R$ matrix

$$
\begin{aligned}
& R(\zeta)_{++}^{++}=R(\zeta)_{--}^{--}=\kappa^{-1}(\zeta) \\
& R(\zeta)_{+-}^{+-}=R(\zeta)_{-+}^{-+}=\kappa^{-1}(\zeta) \frac{(\zeta-1) x}{1-x^{2} \zeta} \\
& R(\zeta)_{+-}^{-+}=R(\zeta)_{-+}^{+-}=\kappa^{-1}(\zeta) \frac{\left(1-x^{2}\right) \zeta^{1 / 2}}{1-x^{2} \zeta}, \quad \kappa(\zeta)=\zeta^{1 / 2} \frac{\left(x^{4} \zeta ; x^{4}\right)_{\infty}\left(x^{2} \zeta^{-1} ; x^{4}\right)_{\infty}}{\left(x^{4} \zeta^{-1} ; x^{4}\right)_{\infty}\left(x^{2} \zeta ; x^{4}\right)_{\infty}}
\end{aligned}
$$

where $(z ; p)_{\infty}=\prod_{n=0}^{\infty}\left(1-z p^{n}\right)$. The $R$ matrix satisfies the Yang-Baxter equation, and $\zeta$ serves as a multiplicative spectral parameter. In particular, the row-to-row transfer matrices $T(\zeta) \equiv T(x ; \zeta)$ commute for different values of $\zeta$ but fixed $x$.

The eigenvectors of the transfer matrix at the infinite lattice are ' $N$-particle' states $\left|\varepsilon_{1} z_{1}, \ldots, \varepsilon_{N} z_{N}\right\rangle$,

$$
T(\zeta)\left|\varepsilon_{1} z_{1}, \ldots, \varepsilon_{N} z_{N}\right\rangle=\prod_{j=1}^{N} \tau\left(z_{j} / \zeta\right)\left|\varepsilon_{1} z_{1}, \ldots, \varepsilon_{N} z_{N}\right\rangle
$$

with $\varepsilon_{j}= \pm \equiv \pm 1$ being the 'charge' of ' $j$ th particle', and $z_{j}$ is the 'rapidity' of ' $j$ th particle',

$$
\left|z_{j}\right|=1
$$

The 'one-particle' eigenvalue function $\tau(z)$ is given by

$$
\tau(z)=z^{1 / 2} \frac{\Theta_{x^{4}}\left(-x^{3} z\right)}{\Theta_{x^{4}}(-x z)},
$$

where $\Theta_{p}(z)=(z ; p)_{\infty}(p / z ; p)_{\infty}(p ; p)_{\infty}$ is a multiplicative form of the $\theta_{1}$ function with the Jacobi parameter $p^{1 / 2}$ :

$$
\Theta_{p}(z)=-2 \mathrm{i} p^{-1 / 8} z^{1 / 2} \theta_{1}(v ; \tau), \quad z=\mathrm{e}^{2 \pi \mathrm{i} v}, \quad \mathrm{e}^{\mathrm{i} \pi \tau}=p^{1 / 2} .
$$

Note that this form of the eigenvalue function is universal for all lattice models based on the $A_{1}$ type $R$ matrices.

We know that in the scaling limit, when we are bringing the system closer to the critical point, simultaneously enlarging the scale, we have to obtain a system of relativistic particles with the states $\left|\varepsilon_{1} \theta_{1}, \ldots, \varepsilon_{N} \theta_{N}\right\rangle$, the Hamiltonian $H$, and momentum operator $P$, such that

$$
\begin{aligned}
& P\left|\varepsilon_{1} \theta_{1}, \ldots, \varepsilon_{N} \theta_{N}\right\rangle=M \sum_{j=1}^{N} \operatorname{sh} \theta_{j}\left|\varepsilon_{1} \theta_{1}, \ldots, \varepsilon_{N} \theta_{N}\right\rangle, \\
& H\left|\varepsilon_{1} \theta_{1}, \ldots, \varepsilon_{N} \theta_{N}\right\rangle=M \sum_{j=1}^{N} \operatorname{ch} \theta_{j}\left|\varepsilon_{1} \theta_{1}, \ldots, \varepsilon_{N} \theta_{N}\right\rangle,
\end{aligned}
$$

with $M$ being the mass of the particles, and $\theta_{j}$ being rapidities. Here the parameter $\theta$ is connected somehow with $z$, and the functions $M \operatorname{ch} \theta$ and $M \operatorname{sh} \theta$ must be related somehow with the function $\tau(z)$. The scattering matrices of the lattice models must as well become the relativistic scattering matrices in the scaling limit.

The scaling limit corresponds to the limit $x \rightarrow 1$, so we set

$$
x=e^{-\epsilon}, \quad \epsilon \rightarrow 0 .
$$


Let us introduce also additive spectral parameters $\gamma$ and $\vartheta$ that survive in the scaling limit:

$$
\begin{gathered}
\zeta=x^{-2 \gamma / \pi}, \quad z=x^{-2 \mathrm{i} \vartheta / \pi} \\
\gamma, \vartheta \in \mathbf{R}, \quad 0 \leq \gamma \leq \pi .
\end{gathered}
$$

It is convenient to pass to the conjugate module in the theta functions. Namely, for the function $\tau(\vartheta)$ we have

$$
\tau(\vartheta)=\frac{\theta_{4}\left(\frac{\mathrm{i} \vartheta}{2 \pi}+\frac{1}{4} ; \frac{\mathrm{i} \pi}{2 \epsilon}\right)}{\theta_{4}\left(\frac{\mathrm{i} \vartheta}{2 \pi}-\frac{1}{4} ; \frac{\mathrm{i} \pi}{2 \epsilon}\right)}=k^{-1 / 2} \operatorname{dn}\left(\frac{\mathbf{K}}{2}-\frac{\mathrm{i} \mathbf{K} \vartheta}{\pi}\right) .
$$

The Jacobi parameter, corresponding to the theta functions is

$$
q=\mathrm{e}^{-\pi^{2} / 2 \epsilon}
$$

We also use the usual designations: $k$ and $k^{\prime}$ are module and conjugate module, $\mathbf{K}$ and $\mathbf{K}^{\prime}$ are half-periods.

The Jacobi parameter $q$ tends to zero in the scaling limit and we can use the standard expansions in $q$ for theta functions. But before doing it let us understand, which values of the spectral parameter correspond to the low-lying excitations which survive at a large scale. The low-lying excitation correspond to the largest in absolute value transfer matrix eigenvalues. The physical values of the spectral parameters $\zeta$ and $z$ satisfy the constraints $1 \leq \zeta \leq x^{-2}$ and $|z|=1$. So we ought to find the maximum of $|\tau(z / \zeta)|^{2}$ in $z$ on the unit circle for fixed $\zeta$ in the interval $1 \leq \zeta \leq x^{-2}$, or, in other words, the maximum of $|\tau(\vartheta+\mathrm{i} \gamma)|^{2}$ in real $\vartheta$ for fixed $\gamma, 0 \leq \gamma \leq \pi$. To do it, note that the function

$$
|\tau(\vartheta+\mathrm{i} \gamma)|^{2}=\frac{\theta_{4}\left(\frac{\mathrm{i} \vartheta}{2 \pi}-\frac{\gamma}{2 \pi}+\frac{1}{4} ; \frac{\mathrm{i} \pi}{2 \epsilon}\right) \theta_{4}\left(\frac{\mathrm{i} \vartheta}{2 \pi}+\frac{\gamma}{2 \pi}-\frac{1}{4} ; \frac{\mathrm{i} \pi}{2 \epsilon}\right)}{\theta_{4}\left(\frac{\mathrm{i} \vartheta}{2 \pi}-\frac{\gamma}{2 \pi}-\frac{1}{4} ; \frac{\mathrm{i} \pi}{2 \epsilon}\right) \theta_{4}\left(\frac{\mathrm{i} \vartheta}{2 \pi}+\frac{\gamma}{2 \pi}+\frac{1}{4} ; \frac{\mathrm{i} \pi}{2 \epsilon}\right)}
$$

of $\vartheta$ can be analytically continued to a doubly periodic function on the complex plane. Then its derivative in $\vartheta$ has two double poles in the periodicity region, and, therefore, has exactly 4 zeros. Using the symmetries of this function it is easy to check that all 4 extremal values are situated at the 'symmetric points' and two of them on the real axis. The maximum is achieved at the point $\vartheta=0$ and the minimum at $\vartheta=\pi^{2} / 2 \epsilon$.

We see that we ought to consider the limit of the elliptic functions $q \rightarrow 0\left(\mathbf{K} \rightarrow \pi / 2, \mathbf{K}^{\prime} \rightarrow \infty\right)$ for the values of $\vartheta$ in any finite interval around 0. Applying to (2.7) the standard limiting formulas

$$
\begin{gathered}
\operatorname{dn} \frac{2 \mathbf{K} \alpha}{\pi}=\frac{\pi}{2 \mathbf{K}}\left[1+4 q \cos 2 \alpha+O\left(q^{2}\right)\right], \\
k^{\prime}=1-8 q+O\left(q^{2}\right), \quad \mathbf{K}=\frac{\pi}{2}\left[1+4 q+O\left(q^{2}\right)\right],
\end{gathered}
$$

we obtain

$$
\tau(\vartheta)=1+4 \mathrm{i} q \operatorname{sh} \vartheta+O\left(q^{2}\right) .
$$

The system can be considered continuous if

$$
q \mathrm{e}^{|\vartheta|} \ll 1 \quad \text { or } \quad-\frac{\pi^{2}}{2(1+\delta) \epsilon}<\vartheta<\frac{\pi^{2}}{2(1+\delta) \epsilon}, \quad \delta>0 .
$$

Let us think that our system lives on the lattice $(a m, b n), m, n \in \mathbf{Z}, a$ and $b$ being lattice parameters. We will think of $a m$ as of the imaginary time it $t^{\prime}$ and of $b n$ as of the space coordinate $x^{\prime}$. Recall that the operator $T(1)$ is the shift operator by $b .{ }^{6}$ So the momentum operator $p^{\prime}$ is given by

$$
\mathrm{e}^{\mathrm{i} p^{\prime}(\vartheta) b} \simeq 1+4 \mathrm{i} q \operatorname{sh} \vartheta
$$

or

$$
p^{\prime}(\vartheta)=\frac{4 q}{b} \operatorname{sh} \vartheta .
$$


The Hamiltonian is given by $\mathrm{e}^{-a H}=T(z)$, and for the one-particle energy we have

$$
\mathrm{e}^{-E^{\prime}(\vartheta) a}=\tau(\vartheta+\mathrm{i} \gamma) \simeq 1+4 \mathrm{i} q \operatorname{sh}(\vartheta+\mathrm{i} \gamma)
$$

or

$$
E^{\prime}(\vartheta)=-\frac{4 \mathrm{i} q}{a} \operatorname{sh}(\vartheta+\mathrm{i} \gamma)=\frac{4 q}{a}(\sin \gamma \operatorname{ch} \vartheta-\mathrm{i} \cos \gamma \operatorname{sh} \vartheta) .
$$

To restore the relativistic spectrum we recall that the initial lattice system is essentially anisotropic. Hence, we need to change coordinates $\left(t^{\prime}, x^{\prime}\right) \rightarrow(t, x)$, so that the spectrum would take the standard form $E(\theta)=$ $M \operatorname{ch} \theta, p(\theta)=M \operatorname{sh} \theta$. It is achieved by the coordinate transformation

$$
\begin{cases}t=t^{\prime} \frac{b}{a} \sin \gamma & =-\mathrm{i} b \sin \gamma \cdot m \\ x=\mathrm{i} t^{\prime} \frac{b}{a} \cos \gamma+x^{\prime} & =+b \cos \gamma \cdot m+b n\end{cases}
$$

and by setting

$$
\theta=\vartheta, \quad M=\frac{4 q}{b} .
$$

Consider now the $S$ matrix. For the six vertex model it is given by ${ }^{6}$

$$
S(z)=-R\left(z^{-1}\right) .
$$

In the scaling limit we obtain

$$
\begin{aligned}
& S(\theta)_{++}^{++}=S(\theta)_{--}^{--}=-S_{0}(\theta), \\
& S(\theta)_{+-}^{+-}=S(\theta)_{-+}^{-+}=-S_{0}(\theta) \frac{\theta}{\mathrm{i} \pi-\theta}, \\
& S(\theta)_{+-}^{-+}=S(\theta)_{-+}^{+-}=-S_{0}(\theta) \frac{\mathrm{i} \pi}{\mathrm{i} \pi-\theta}, \quad S_{0}(\theta)=\frac{\Gamma\left(1+\frac{\mathrm{i} \theta}{2 \pi}\right) \Gamma\left(\frac{1}{2}-\frac{\mathrm{i} \theta}{2 \pi}\right)}{\Gamma\left(1-\frac{\mathrm{i} \theta}{2 \pi}\right) \Gamma\left(\frac{1}{2}+\frac{\mathrm{i} \theta}{2 \pi}\right)} .
\end{aligned}
$$

This $S$ matrix coincides with the critical sine-Gordon $S$ matrix. ${ }^{a}$

The sine-Gordon model is the model with the action

$$
\mathcal{A}=\int d^{2} x\left(\frac{1}{16 \pi}\left(\partial_{\mu} \varphi\right)^{2}+\frac{\lambda}{\beta^{2}} \cos \beta \varphi\right) .
$$

The dimensionality of the constant $\lambda$ is $M^{2-2 \beta^{2}}$. For $0<\beta<1$ the term $\cos \beta \varphi$ is a relevant perturbation, and the theory is superrenormalizable. For $\beta>1$ the perturbation is irrelevant, and the properties of the theory are completely unknown. The critical sine-Gordon theory corresponds to the marginal value $\beta=1$. In fact, dimensional transmutation takes place at this point, and the theory is an integrable theory of massive interactive charged solitons. $S(\theta)$ is the pair soliton-soliton $S$ matrix.

\section{Free Field Representation and Conjugate Zero Mode}

Now let us turn to the calculation of form factors. We start from the free field representation of Ref. 6 .

Consider the Heisenberg algebra

$$
[P, Q]=-\mathrm{i}, \quad\left[a_{m}, a_{n}\right]=\delta_{m+n, 0} \frac{[2 m]_{x}[m]_{x}}{m} \quad\left([s]_{x}=\frac{x^{s}-x^{-s}}{x-x^{-1}}\right)
$$

The spaces $\mathcal{H}_{i}, i=0,1$ are generated by the operators $a_{-n}, n>0$, and $\exp ( \pm \mathrm{i} \sqrt{2} Q)$ from the vacuum vectors $|0\rangle^{(i)}$, defined as

$$
\begin{aligned}
a_{n}|0\rangle^{(i)} & =0, \quad n>0, \\
P|0\rangle^{(i)} & =\frac{i}{\sqrt{2}}|0\rangle^{(i)} .
\end{aligned}
$$

$a$ In the case of the six vertex model we do not need the duality transformation here. ${ }^{10}$ 
We also shall use the notation $|p\rangle$ for the vector

$$
|p\rangle=\mathrm{e}^{\mathrm{i} p Q}|0\rangle^{(0)}, \quad P|p\rangle=p|p\rangle .
$$

The vertex operators $V_{\varepsilon}^{(1-i, i)}(\zeta), \tilde{V}_{\varepsilon}^{(1-i, i)}(z): \mathcal{H}_{i} \rightarrow \mathcal{H}_{1-i}$ are defined as ${ }^{6 b}$

$$
\begin{aligned}
V_{+}^{(1-i, i)}(\zeta) & =G^{1 / 2} x^{3 / 4}: \mathrm{e}^{\mathrm{i} \phi(\zeta)}: \\
V_{-}^{(1-i, i)}(\zeta) & =G^{1 / 2} x^{3 / 4} \oint_{C_{1}} \frac{d v}{2 \pi \mathrm{i}} F_{1}(v, \zeta): \mathrm{e}^{\mathrm{i} \phi(\zeta)-\mathrm{i} \phi\left(x^{-1} v\right)-\mathrm{i} \phi(x v)}: \\
\tilde{V}_{+}^{(1-i, i)}(z) & =G^{-1 / 2} x^{3 / 4} z^{1 / 2}: \mathrm{e}^{\mathrm{i} \tilde{\phi}(z)}: \\
\tilde{V}_{-}^{(1-i, i)}(z) & =G^{-1 / 2} x^{3 / 4} z^{1 / 2} \oint_{C_{2}} \frac{d w}{2 \pi \mathrm{i}} F_{2}(w, z): \mathrm{e}^{\mathrm{i} \tilde{\phi}(z)-\mathrm{i} \tilde{\phi}\left(x^{-1} w\right)-\mathrm{i} \tilde{\phi}(x w)}:
\end{aligned}
$$

where

$$
G=\frac{\left(x^{2} ; x^{4}\right)_{\infty}}{\left(x^{4} ; x^{4}\right)_{\infty}}, \quad F_{1}(v, \zeta)=\frac{\left(1-x^{2}\right) v \zeta^{1 / 2}}{x\left(v-x^{-1} \zeta\right)(v-x \zeta)}, \quad F_{2}(w, z)=\frac{\left(1-x^{2}\right) z^{1 / 2}}{x\left(w-x^{-1} z\right)(w-x z)}
$$

and

$$
\begin{aligned}
& \phi(\zeta)=\frac{1}{\sqrt{2}}\left(Q-\mathrm{i} P \log \left(x^{3} \zeta\right)\right)+\sum_{n \neq 0} \frac{a_{n}}{\mathrm{i}[2 n]_{x}} x^{|n| / 2} \zeta^{-n}, \\
& \tilde{\phi}(z)=-\frac{1}{\sqrt{2}}\left(Q-\mathrm{i} P \log \left(x^{3} z\right)\right)-\sum_{n \neq 0}(-1)^{n} \frac{a_{n}}{\mathrm{i}[2 n]_{x}} x^{-|n| / 2} z^{-n} .
\end{aligned}
$$

The normal ordering operation $: \ldots .:$ places $P$ to the right of $Q$, and $a_{n}$ to the right of $a_{-n}, n>0$. The contours $C_{1}$ and $C_{2}$ encircle the point 0 so that $x \zeta$ is inside and $x^{-1} \zeta$ is outside $C_{1}$, and $x^{-1} z$ is inside and $x z$ is outside $C_{2}$.

The form factors of local operators $O$ are given by ${ }^{6}$

$$
\left\langle 0|O| \varepsilon_{1} \zeta_{1} \ldots \varepsilon_{N} \zeta_{N}\right\rangle_{\text {in }}^{(i)}=\frac{1}{\chi_{i}\left(x^{4}\right)} \operatorname{Tr}_{\mathcal{H}_{i}}\left(x^{4 D^{(i)}} \hat{O}^{\left(i, i^{\prime}\right)} \tilde{V}_{\varepsilon_{N}}^{\left(i^{\prime}, 1-i^{\prime}\right)}\left(z_{N}\right) \ldots \tilde{V}_{\varepsilon_{1}}^{(1-i, i)}\left(z_{1}\right)\right),
$$

Here

$$
D^{(i)}=\frac{P^{2}}{2}-\frac{P}{2 \sqrt{2}}+\sum_{n=1}^{\infty} \frac{n^{2}}{[2 n]_{x}[n]_{x}} a_{-n} a_{n}
$$

is the shift operator

$$
\left[D^{(i)}, V_{\varepsilon}^{(1-i, i)}(\zeta)\right]=\zeta \frac{d}{d \zeta} V_{\varepsilon}^{(1-i, i)}(\zeta), \quad\left[D^{(i)}, \tilde{V}_{\varepsilon}^{(1-i, i)}(z)\right]=z \frac{d}{d z} \tilde{V}_{\varepsilon}^{(1-i, i)}(z),
$$

and $\chi_{i}(t)$ is the character of $\mathcal{H}_{i}$,

$$
\chi_{i}(t)=\operatorname{Tr}_{\mathcal{H}_{i}} t^{D^{(i)}}=\left(t^{1 / 2} ; t\right)_{\infty}^{-1} .
$$

The operator $\hat{O}^{\left(i, i^{\prime}\right)}$ is determined by the operator $O$ and consists of the operators $V_{\varepsilon}^{(1-i, i)}(\zeta)$. We shall consider here an important example of the polarization operator $\sigma^{z}=\left(\begin{array}{cc}1 & 0 \\ 0 & -1\end{array}\right)$, inserted into one of the vertical links of the lattice. The respective operator ${\widehat{\left(\sigma^{z}\right)}}^{(i, i)}$ is given by

$$
{\widehat{\left(\sigma^{z}\right)}}^{(i, i)}=-\sum_{\varepsilon} \varepsilon V_{-\varepsilon}^{(i, 1-i)}\left(x^{-2} \zeta\right) V_{\varepsilon}^{(1-i, i)}(\zeta)
$$

${ }^{b}$ In terms of Ref. $6 V_{\varepsilon}^{(1-i, i)}(\zeta) \sim \Phi_{-\varepsilon}^{(1-i, i)}\left(\zeta^{1 / 2}\right), \tilde{V}_{\varepsilon}^{(1-i, i)}(z) \sim \Psi_{-\varepsilon}^{*(1-i, i)}(z)$. 
Consider an arbitrary trace

$$
T_{N}^{(i)}=\frac{1}{\chi_{i}\left(x^{4}\right)} \operatorname{Tr}_{\mathcal{H}_{i}}\left(x^{4 D^{(i)}}: \mathrm{e}^{\phi_{N}}: \ldots: \mathrm{e}^{\phi_{1}}:\right)
$$

where

$$
: \mathrm{e}^{\phi_{j}}:=\mathrm{e}^{\alpha_{j} Q} \mathrm{e}^{\epsilon \beta_{j} P} \exp \left(\sum_{n=1}^{\infty} A_{n} a_{-n}\right) \exp \left(\sum_{n=1}^{\infty} A_{-n} a_{n}\right) .
$$

It is easy to check that the trace can be factorized,

$$
T_{N}^{(i)}=H_{N}^{(i)} F_{N},
$$

into the contribution of the zero mode $(Q, P), H_{N}^{(i)}$, and the contribution of the oscillators $a_{n}, F_{N}$. The oscillators contribution is given by

$$
\begin{aligned}
F_{N} & =\prod_{j} C_{j} \prod_{j<j^{\prime}} F_{j j^{\prime}} \\
\log C_{j} & =\frac{1}{\chi_{*}\left(x^{4}\right)} \operatorname{Tr}_{*}\left(x^{4 D_{*}} \phi_{+, j} \phi_{-, j}\right) \\
\log F_{j j^{\prime}} & =\frac{1}{\chi_{*}\left(x^{4}\right)} \operatorname{Tr}_{*}\left(x^{4 D_{*}} \phi_{* j} \phi_{* j^{\prime}}\right), \\
\chi_{*}(t) & =\operatorname{Tr}_{*}\left(t^{D_{*}}\right)
\end{aligned}
$$

Here asterisks mean that the contribution of oscillators is only taken into account; $\phi_{+}$and $\phi_{-}$are contributions into $\phi$ from $a_{-n}$ and $a_{n}$ respectively $(n>0)$.

The zero mode contribution is the most interesting part. Let us write it down explicitly. It is only nonzero for $\sum_{j} \alpha_{j}=0$. Let us introduce the notations

$$
A=\sum_{j<j^{\prime}}^{N} \alpha_{j} \beta_{j^{\prime}}, \quad B=\sum_{j=1}^{N} \beta_{j} .
$$

Then

$$
\begin{aligned}
H_{N}^{(i)} \chi_{0}\left(x^{4}\right) & =\operatorname{Tr}_{0}^{(i)}\left(x^{4 D_{0}^{(i)}} \mathrm{e}^{\alpha_{N} Q} \mathrm{e}^{\epsilon \beta_{N} P} \ldots \mathrm{e}^{\alpha_{1} Q} \mathrm{e}^{\epsilon \beta_{1} P}\right) \\
& =\sum_{n \in \mathbf{Z}+i / 2}\left\langle n \sqrt{2}\left|\mathrm{e}^{-4 \epsilon\left(\frac{P^{2}}{2}-\frac{P}{2 \sqrt{2}}\right)} \mathrm{e}^{\alpha_{N} Q} \mathrm{e}^{\epsilon \beta_{N} P} \ldots \mathrm{e}^{\alpha_{1} Q} \mathrm{e}^{\epsilon \beta_{1} P}\right| n \sqrt{2}\right\rangle \\
& =\mathrm{e}^{-\mathrm{i} \epsilon A} \sum_{n \in \mathbf{Z}+i / 2} \exp \left(-4 \epsilon\left(n^{2}-\frac{n}{2}\right)+\epsilon n \sqrt{2} \sum_{j} \beta_{j}\right)=\mathrm{e}^{-\mathrm{i} \epsilon A} \theta_{3-i}\left(-\mathrm{i} \epsilon \frac{2+\sqrt{2} B}{2 \pi} ; \mathrm{i} \frac{4 \epsilon}{\pi}\right) \\
& =\frac{1}{2} \sqrt{\frac{\pi}{\epsilon}} \mathrm{e}^{-\mathrm{i} \epsilon A} \exp \left[4 \epsilon\left(\frac{1}{4}+\frac{B}{4 \sqrt{2}}\right)^{2}\right] \theta_{3+i}\left(\frac{1}{4}+\frac{B}{4 \sqrt{2}} ; \mathrm{i} \frac{\pi}{4 \epsilon}\right),
\end{aligned}
$$

where the subscript 0 means the contribution of the zero mode. We passed to a conjugate module in the last equality. The most interesting feature of this expression is that we can rewrite the last theta function as a trace of an operator. Expanding it in the series, we can easily check that

$$
\begin{aligned}
H_{N}^{(i)} \chi_{0}\left(x^{4}\right)= & \frac{1}{2} \sqrt{\frac{\pi}{\epsilon}} \exp \left[4 \epsilon\left(\frac{1}{4}+\frac{B}{4 \sqrt{2}}\right)^{2}\right] \\
& \times \sum_{n \in \mathbf{Z}}\left\langle\frac{\pi n}{2 \sqrt{2}}\left|\mathrm{e}^{-\frac{4}{\epsilon} \frac{P^{\prime 2}}{2}+\mathrm{i}(-1)^{i} \sqrt{2} P^{\prime}} \mathrm{e}^{-\mathrm{i} \epsilon \alpha_{N} Q^{\prime}} \mathrm{e}^{\mathrm{i} \beta_{N} P^{\prime}} \ldots \mathrm{e}^{-\mathrm{i} \epsilon \alpha_{1} Q^{\prime}} \mathrm{e}^{\mathrm{i} \beta_{1} P^{\prime}}\right| \frac{\pi n}{2 \sqrt{2}}\right\rangle^{\prime} .
\end{aligned}
$$


Here $P^{\prime}$ and $Q^{\prime}$ is a new pair of canonically conjugate operators and $|p\rangle^{\prime}$ designates the eigenvector of $P^{\prime}$ :

$$
\left[P^{\prime}, Q^{\prime}\right]=-\mathrm{i}, \quad P^{\prime}|p\rangle^{\prime}=p|p\rangle^{\prime}, \quad|p\rangle^{\prime}=\mathrm{e}^{\mathrm{i} p Q}|0\rangle^{\prime} .
$$

Naively, the term with $n=0$ only survives in the limit $\epsilon \rightarrow 0$. But as we shall see below in the calculation of the form factors of $\sigma^{z}$ this term vanishes, so that the terms with $n= \pm 1$ become leading.

Note also that we can substitute $P \rightarrow \mathrm{i} P^{\prime} / \epsilon$ and $Q \rightarrow-\mathrm{i} \epsilon Q^{\prime}$ directly in the vertex operators. This substitution does not affect the commutation relations of the vertex operators, but the summation rule over the momentum values changes drastically and the operator $D^{(i)}$ must be substituted by

$$
D^{\prime(i)}=\epsilon^{-2}\left(\frac{P^{\prime 2}}{2}-\mathrm{i} \frac{(-)^{i} P^{\prime}}{2 \sqrt{2}}\right)+\sum_{n=1}^{\infty} \frac{n^{2}}{[2 n]_{x}[n]_{x}} a_{-n} a_{n} .
$$

The explicit form of the nonzero 'two-point' traces is

$$
\begin{aligned}
& -\log F_{11}\left(\gamma_{1}-\gamma_{2}\right)=\frac{1}{\chi_{*}\left(x^{4}\right)} \operatorname{Tr}_{*}\left(x^{4 D_{*}} \phi_{*}\left(\zeta_{1}\right) \phi_{*}\left(\zeta_{2}\right)\right)=\sum_{n=1}^{\infty} \frac{\mathrm{e}^{-\epsilon n}}{n} \frac{\operatorname{sh} \epsilon n}{\operatorname{sh}^{2} 2 \epsilon n} \operatorname{ch} 2 \epsilon n\left(1-\frac{\gamma_{1}-\gamma_{2}}{\pi}\right), \\
& -\log F_{22}\left(\theta_{1}-\theta_{2}\right)=\frac{1}{\chi_{*}\left(x^{4}\right)} \operatorname{Tr}_{*}\left(x^{4 D_{*}} \tilde{\phi}_{*}\left(z_{1}\right) \tilde{\phi}_{*}\left(z_{2}\right)\right)=\sum_{n=1}^{\infty} \frac{\mathrm{e}^{\epsilon n}}{n} \frac{\operatorname{sh} \epsilon n}{\operatorname{sh}^{2} 2 \epsilon n} \operatorname{ch} 2 \epsilon n\left(1-\mathrm{i} \frac{\theta_{1}-\theta_{2}}{\pi}\right), \\
& -\log F_{12}\left(\gamma_{1}-\mathrm{i} \theta_{2}\right)=\frac{1}{\chi_{*}\left(x^{4}\right)} \operatorname{Tr}_{*}\left(x^{4 D_{*}} \phi_{*}\left(\zeta_{1}\right) \tilde{\phi}_{*}\left(z_{2}\right)\right)=\sum_{n=1}^{\infty} \frac{(-1)^{n-1}}{n} \frac{\operatorname{sh} \epsilon n}{\operatorname{sh}^{2} 2 \epsilon n} \operatorname{ch} 2 \epsilon n\left(1-\frac{\gamma_{1}-\mathrm{i} \theta_{2}}{\pi}\right) .
\end{aligned}
$$

Similarly, the traces of the form $\chi_{*}^{-1}\left(x^{4}\right) \operatorname{Tr}_{*}\left(x^{4 D_{*}} \phi_{+} \phi_{-}\right)$are

$$
\begin{aligned}
-\log \left[x^{-3 / 4} G^{-1 / 2} C_{+}\right] & =\sum_{n=1}^{\infty} \frac{\mathrm{e}^{-3 \epsilon n} \operatorname{sh} \epsilon n}{2 n \operatorname{sh}^{2} 2 \epsilon n}, \\
-\log \left[x^{-3 / 4} G^{-1 / 2} F_{1}^{-1}(v, \zeta) C_{-}(\gamma, \mu)\right] & =\sum_{n=1}^{\infty} \frac{\mathrm{e}^{-3 \epsilon n} \operatorname{sh} \epsilon n}{2 n \operatorname{sh}^{2} 2 \epsilon n}\left[-4\left(\operatorname{ch} 2 \epsilon n \frac{\mu}{\pi}-1\right) \operatorname{ch} \epsilon n+(2 \operatorname{ch} \epsilon n-1)^{2}\right], \\
-\log \left[x^{-3 / 4} G^{1 / 2} \tilde{C}_{+}\right] & =\sum_{n=1}^{\infty} \frac{\mathrm{e}^{-\epsilon n} \operatorname{sh} \epsilon n}{2 n \operatorname{sh}^{2} 2 \epsilon n}, \\
-\log \left[x^{-3 / 4} G^{1 / 2} F_{2}^{-1}(w, z) \tilde{C}_{-}(\theta, \nu)\right] & =\sum_{n=1}^{\infty} \frac{\mathrm{e}^{-\epsilon n} \operatorname{sh} \epsilon n}{2 n \operatorname{sh}^{2} 2 \epsilon n}\left[-4\left(\operatorname{ch} 2 \epsilon n \frac{\mathrm{i} \nu}{\pi}-1\right) \operatorname{ch} \epsilon n+(2 \operatorname{ch} \epsilon n-1)^{2}\right], \\
v & =\mathrm{e}^{\frac{2 \epsilon}{\pi}(\gamma+\mu)}, \quad w=\mathrm{e}^{\frac{2 \mathrm{i} \epsilon}{\pi}(\theta+\nu)} .
\end{aligned}
$$

It is convenient also to introduce the functions

$$
\begin{aligned}
\bar{F}_{11}(\gamma)=F_{11}^{-1}(\gamma-\pi / 2) F_{11}^{-1}(\gamma+\pi / 2), & & \overline{\bar{F}}_{11}(\gamma)=\bar{F}_{11}^{-1}(\gamma-\pi / 2) \bar{F}_{11}^{-1}(\gamma+\pi / 2), \\
\bar{F}_{12}(\gamma)=F_{12}^{-1}(\gamma-\pi / 2) F_{12}^{-1}(\gamma+\pi / 2), & & \overline{\bar{F}}_{12}(\gamma)=\bar{F}_{12}^{-1}(\gamma-\pi / 2) \bar{F}_{12}^{-1}(\gamma+\pi / 2), \\
\bar{F}_{22}(\theta)=F_{22}^{-1}(\theta+\mathrm{i} \pi / 2) F_{22}^{-1}(\theta-\mathrm{i} \pi / 2), & & \overline{\bar{F}}_{22}(\theta)=\bar{F}_{22}^{-1}(\theta+\mathrm{i} \pi / 2) \bar{F}_{22}^{-1}(\theta-\mathrm{i} \pi / 2),
\end{aligned}
$$

All traces of the vertex operators can be written in terms of the functions (3.12-3.14). To do it we need to write a function $C_{\varepsilon}, z_{j}^{1 / 2} \tilde{C}_{\varepsilon}$ for each $V_{\varepsilon}\left(\zeta_{j}\right), \tilde{V}_{\varepsilon}\left(z_{j}\right)$, a function $F_{11}\left(\gamma_{j}-\gamma_{j^{\prime}}\right)$ for each pair $V_{+}\left(\zeta_{j}\right)$ and $V_{+}\left(\zeta_{j^{\prime}}\right), j>j^{\prime}$, a function $F_{11}\left(\gamma_{j}-\gamma_{j^{\prime}}\right) \bar{F}_{11}\left(\gamma_{j}-\gamma_{j^{\prime}}-\mu_{j^{\prime}}\right)$ for each pair $V_{+}\left(\zeta_{j}\right)$ and $V_{-}\left(\zeta_{j^{\prime}}\right), j>j^{\prime}$, etc., then to write the overall coefficient from the zero mode, and finally to integrate over all variables $\mu_{j}$ and $\nu_{j}$.

\section{Scaling Limit of Form Factors}

Consider the scaling limit of the functions (3.12). Applying the formula

$$
\sum_{n=1}^{\infty}\left(\frac{a_{2}}{\epsilon n^{2}}+\frac{a_{1} \mathrm{e}^{-\epsilon n}}{n}+\epsilon f(\epsilon n)\right)=\frac{\pi^{2}}{6 \epsilon} a_{2}-a_{1} \log \epsilon+\int_{0}^{\infty} d \eta f(\eta)+O(\epsilon)
$$


for $f(\eta)$ being finite as $\eta \rightarrow 0$, we easily obtain

$$
\begin{aligned}
& F_{11}(\gamma)=\epsilon^{-1 / 4} q^{1 / 12}\left(f_{11}(\gamma)+O(\epsilon)\right), \\
& F_{22}(\theta)=\epsilon^{1 / 4} q^{1 / 12}\left(f_{22}(\theta)+O(\epsilon)\right), \\
& F_{12}(\gamma)=q^{1 / 24}\left(\exp \frac{\epsilon}{4}\left[\left(1-\frac{\gamma}{\pi}\right)^{2}-\frac{7}{12}\right]+O\left(\epsilon^{\infty}\right)\right),
\end{aligned}
$$

with

$$
\begin{aligned}
& -\log f_{11}(\gamma)=\int_{0}^{\infty} \frac{d \eta}{\eta}\left[\mathrm{e}^{-\eta} \frac{\operatorname{sh} \eta}{\operatorname{sh}^{2} 2 \eta} \operatorname{ch} 2 \eta\left(1-\frac{\gamma}{\pi}\right)-\frac{1}{4 \eta}+\frac{1}{4} \mathrm{e}^{-\eta}\right], \\
& -\log f_{22}(\theta)=\int_{0}^{\infty} \frac{d \eta}{\eta}\left[\mathrm{e}^{\eta} \frac{\operatorname{sh} \eta}{\operatorname{sh}^{2} 2 \eta} \operatorname{ch} 2 \eta\left(1-\mathrm{i} \frac{\theta}{\pi}\right)-\frac{1}{4 \eta}-\frac{1}{4} \mathrm{e}^{-\eta}\right] .
\end{aligned}
$$

The expression for $F_{12}$ demands a more detailed consideration. It is easy to check, that the formula is correct up to all orders in $\epsilon$, because in the decomposition of the summand in $\epsilon$ the odd powers of $n$ vanish. And it is easy to check that $\sum_{n=1}^{\infty}(-1)^{n-1} n^{2 p}=0, p \geq 1$. However, we need a more strong (and more rigorous) estimation than $O\left(\epsilon^{\infty}\right)$. We can give it for the ratio

$$
\frac{\bar{F}_{12}(\gamma+\pi / 2)}{\bar{F}_{12}(\gamma-\pi / 2)}=\frac{\Theta_{x^{4}}(-x y)}{\Theta_{x^{4}}\left(-x^{3} y\right)}=y^{1 / 2}[1+O(q)], \quad y=x^{2(1-\gamma / \pi)} .
$$

The last equality follows from the earlier consideration of the function $\tau(z)$. We shall see that this estimation is strong enough to find the form factors of $\sigma^{z}$.

For $C_{ \pm}, \tilde{C}_{ \pm}$we find

$$
\begin{aligned}
C_{+} & \simeq \epsilon^{-1 / 8} q^{1 / 24} c_{+}, \\
v C_{-}(\gamma, \mu) & \simeq \epsilon^{-1 / 8} q^{1 / 24} c_{-} \frac{\pi}{2 \epsilon} f_{1}(\mu) \mathrm{e}^{\epsilon \gamma / \pi}, \\
\tilde{C}_{+} & \simeq \epsilon^{-3 / 8} q^{1 / 24} \tilde{c}_{+}, \\
w \tilde{C}_{-}(\theta, \nu) & \simeq \epsilon^{-3 / 8} q^{1 / 24} \tilde{c}_{-} \frac{\pi}{2 \epsilon} f_{2}(\nu)
\end{aligned}
$$

with

$$
\begin{aligned}
& \log c_{+}=-\frac{1}{2} \int_{0}^{\infty} \frac{d \eta}{\eta}\left(\mathrm{e}^{-3 \eta} \frac{\operatorname{sh} \eta}{\operatorname{sh}^{2} 2 \eta}-\frac{1}{4 \eta}+\frac{1}{4} \mathrm{e}^{-\eta}+\frac{\mathrm{e}^{-\eta}}{2 \operatorname{ch} \eta}\right), \\
& \log c_{-}=-\frac{1}{2} \int_{0}^{\infty} \frac{d \eta}{\eta}\left(\mathrm{e}^{-3 \eta} \frac{\operatorname{sh} \eta}{\operatorname{sh}^{2} 2 \eta}(2 \operatorname{ch} \eta-1)^{2}-\frac{1}{4 \eta}+\frac{1}{4} \mathrm{e}^{-\eta}+\frac{\mathrm{e}^{-\eta}}{2 \operatorname{ch} \eta}\right), \\
& \log \tilde{c}_{+}=-\frac{1}{2} \int_{0}^{\infty} \frac{d \eta}{\eta}\left(\mathrm{e}^{-\eta} \frac{\operatorname{sh} \eta}{\operatorname{sh}^{2} 2 \eta}-\frac{1}{4 \eta}+\frac{1}{4} \mathrm{e}^{-\eta}-\frac{\mathrm{e}^{-\eta}}{2 \operatorname{ch} \eta}\right), \\
& \log \tilde{c}_{-}=-\frac{1}{2} \int_{0}^{\infty} \frac{d \eta}{\eta}\left(\mathrm{e}^{-\eta} \frac{\operatorname{sh} \eta}{\operatorname{sh}^{2} 2 \eta}(2 \operatorname{ch} \eta-1)^{2}-\frac{1}{4 \eta}+\frac{1}{4} \mathrm{e}^{-\eta}-\frac{\mathrm{e}^{-\eta}}{2 \operatorname{ch} \eta}\right), \\
& f_{1}(\mu)=\frac{\pi}{(\mu-\pi / 2)(\mu+\pi / 2)} \exp \int_{0}^{\infty} \frac{d \eta}{\eta} \frac{\mathrm{e}^{-3 \eta}}{\operatorname{sh} 2 \eta}\left(\operatorname{ch} 2 \eta \frac{\mu}{\pi}-1\right) \\
& f_{2}(\nu)=-\frac{\pi}{(\nu+\mathrm{i} \pi / 2)(\nu-\mathrm{i} \pi / 2)} \exp \int_{0}^{\infty} \frac{d \eta}{\eta} \frac{\mathrm{e}^{-\eta}}{\operatorname{sh} 2 \eta}\left(\operatorname{ch} 2 \eta \frac{\mathrm{i} \nu}{\pi}-1\right) .
\end{aligned}
$$

All equalities in (4.4) are up to $O(\epsilon)$, but the expression for $C_{-}$includes the precise dependence on $\gamma$, which will be important later.

We need to make one more remark. The exact formulas for form factors on the lattice contain integrations over closed paths encircling zero. It means that we must integrate over $\mu$ and $\nu$ along to infinite contours. But at $|\mu|,|\nu| \sim 1 / \epsilon$ the formulas (4.1-4.5) is no more valid. We should analyze its contribution. We can argue in the following way. It is easy to check that

$$
\begin{aligned}
f_{11}(\mathrm{i} \theta) & \sim|\theta|^{-1 / 4} \mathrm{e}^{|\theta| / 4}, & f_{1}(\mathrm{i} \theta) & \sim|\theta|^{-1 / 2} \mathrm{e}^{-|\theta| / 2}, \\
f_{22}(\theta) & \sim|\theta|^{1 / 4} \mathrm{e}^{|\theta| / 4}, & f_{2}(\theta) & \sim|\theta|^{-3 / 2} \mathrm{e}^{-|\theta| / 2}
\end{aligned}
$$


for large real $\theta$. Therefore for large imaginary $\mu$ or large real $\nu$ a factor $\sim f_{1}(\mu) f_{11}^{-2}(\mu) \sim \mathrm{e}^{-|\mu|}$ or $\sim f_{2}(\nu) f_{22}^{-2}(\nu) \sim|\nu|^{-2} \mathrm{e}^{-|\nu|}$ appears. We shall see that the zero mode gives the factors $\sim \mathrm{e}^{|\mu|}, \mathrm{e}^{|\nu|}$ in the case of $\sigma^{z}$. So the contribution of $\nu_{j} \sim 1 / \epsilon$ is negligible, but the contribution of $\mu \sim 1 / \epsilon$ must be taken into account. Nevertheless, as we shall discuss later, the correct answer can be extracted from the approximation for small $\mu$.

At last, we define the states

$$
\left|\varepsilon_{1} \theta_{1}, \ldots, \varepsilon_{N} \theta_{N}\right\rangle_{\text {in }}^{(i)}=\left(\frac{2 \epsilon}{\pi}\right)^{N / 2}\left|\varepsilon_{1} z_{1}, \ldots, \varepsilon_{N} z_{N}\right\rangle_{\text {in }}^{(i)}
$$

so that it would have the natural for two-dimensional field theory normalization:

$$
\frac{1}{N !} \int \frac{d^{N} \theta}{(2 \pi)^{N}}{ }_{\text {in }}^{(i)}\left\langle\varepsilon_{1} \theta_{1}^{\prime}, \ldots, \varepsilon_{N} \theta_{N}^{\prime} \mid \varepsilon_{1} \theta_{1}, \ldots, \varepsilon_{N} \theta_{N}\right\rangle_{\text {in }}^{(i)}=1 .
$$

Now we are ready to calculate of the form factors of $\sigma^{z}$ in the scaling limit. The form factors of $\sigma^{z}$ read precisely

$$
\left\langle 0\left|\sigma^{z}\right| \varepsilon_{1} \theta_{1}, \ldots, \varepsilon_{2 n} \theta_{2 n}\right\rangle_{\mathrm{in}}^{(i)}=-\int_{C_{1}^{\prime}} \frac{d \mu}{2 \pi \mathrm{i}} \int_{C_{2}^{\prime}} \frac{d^{n} \nu}{(2 \pi)^{n}} \sum_{\alpha= \pm} \alpha H_{-\alpha \alpha}^{(i)}(\gamma, \mu ; \varepsilon, \theta, \nu) F_{-\alpha \alpha}(\gamma, \mu ; \varepsilon, \theta, \nu),
$$

where $H$ and $F$ are contributions of the zero mode and the oscillators respectively. Let us begin with the oscillators.

The contour $C_{1}^{\prime}$ goes from $-\mathrm{i} \infty$ to $+\mathrm{i} \infty$ to the right of $-\frac{1}{2} \pi$ and to the left of $\frac{1}{2} \pi$, and the contour $C_{2}^{\prime}$ goes from $-\infty$ to $+\infty$ above $\frac{\mathrm{i}}{2} \pi$ and below $-\frac{\mathrm{i}}{2} \pi$ (with an inflection).

The oscillators contribution factorizes as follows:

$$
F_{-\alpha \alpha}(\gamma, \mu ; \varepsilon, \theta, \nu)=\frac{2 \epsilon}{\pi} F_{-\alpha \alpha}(\mu) F(\varepsilon, \theta, \nu) S_{-\alpha \alpha}(\gamma, \mu ; \varepsilon, \theta, \nu) .
$$

Here $F_{-\alpha \alpha}(\mu)$ is the $F$ function for the vacuum expectation of $\sigma^{z}$ :

$$
\begin{aligned}
& F_{-+}(\mu)=\frac{2 \epsilon}{\pi} C_{-}(\gamma+\pi, \mu) C_{+} F_{11}(\pi) \bar{F}_{11}(\mu+\pi), \\
& F_{+-}(\mu)=\frac{2 \epsilon}{\pi} C_{+} C_{-}(\gamma, \mu) F_{11}(\pi) \bar{F}_{11}(\pi-\mu) .
\end{aligned}
$$

¿From the evident property $F_{11}(\gamma+2 \pi)=F_{11}(-\gamma)$ we obtain

$$
\frac{F_{+-}(\mu)}{F_{-+}(\mu)}=\frac{C_{-}(\gamma)}{C_{-}(\gamma+\pi)}=x=\mathrm{e}^{-\epsilon} .
$$

Note that this result is precise. Up to $O(\epsilon)$ the quantities $F_{-+}(\mu)$ and $F_{+-}(\mu)$ coincide and

$$
F_{-+}(\mu) \simeq c_{+} c_{-} f_{1}(\mu) f_{11}(\pi) \bar{f}_{11}(\mu+\pi) .
$$

The function $F(\varepsilon, \theta, \nu)$ is the factor that only depends on the asymptotic states rather than on the local operator:

$$
\begin{aligned}
F(\varepsilon, \theta, \nu)= & \left(\frac{2 \epsilon}{\pi}\right)^{2 n} \mathrm{e}^{\mathrm{i} \frac{\epsilon}{\pi} \sum \theta_{j}} \tilde{C}_{+}^{n} \prod_{j \in N_{-}} \tilde{C}_{-}\left(\theta_{j}, \nu_{j}\right) \prod_{j<j^{\prime}} F_{22}\left(\theta_{j^{\prime}}-\theta_{j}\right) \\
& \times \prod_{j<j^{\prime}, j^{\prime} \in N_{-}} \bar{F}_{22}\left(\theta_{j^{\prime}}-\theta_{j}+\nu_{j^{\prime}}\right) \prod_{j<j^{\prime}, j \in N_{-}} \bar{F}_{22}\left(\theta_{j^{\prime}}-\theta_{j}-\nu_{j}\right) \prod_{j<j^{\prime} \in N_{-}} \overline{\bar{F}}_{22}\left(\theta_{j^{\prime}}-\theta_{j}+\nu_{j^{\prime}}-\nu_{j}\right) \\
\simeq & \left(\frac{2}{\pi}\right)^{n} \tilde{c}_{+}^{n} \tilde{c}_{-}^{n} \prod_{j \in N_{-}} f_{2}\left(\theta_{j}, \nu_{j}\right) \prod_{j<j^{\prime}} f_{22}\left(\theta_{j^{\prime}}-\theta_{j}\right) \\
& \times \prod_{j<j^{\prime}, j^{\prime} \in N_{-}} \bar{f}_{22}\left(\theta_{j^{\prime}}-\theta_{j}+\nu_{j^{\prime}}\right) \prod_{j<j^{\prime}, j \in N_{-}} \bar{f}_{22}\left(\theta_{j^{\prime}}-\theta_{j}-\nu_{j}\right) \\
& \times \prod_{j<j^{\prime} \in N_{-}} \overline{\bar{f}}_{22}\left(\theta_{j^{\prime}}-\theta_{j}+\nu_{j^{\prime}}-\nu_{j}\right)
\end{aligned}
$$


with $N_{ \pm}=\left\{j \mid \varepsilon_{j}= \pm\right\}$. From the requirement $\sum_{j} \alpha_{j}=0$ we have $\sum_{j} \varepsilon_{j}=0$ or $\# N_{+}=\# N_{-}$.

The third factor $S_{-\alpha \alpha}(\gamma, \mu ; \varepsilon, \theta, \nu)$ is given by

$$
\begin{aligned}
S_{-+}(\gamma, \mu ; \varepsilon, \theta, \nu)= & \prod_{j} F_{12}\left(\gamma-\mathrm{i} \theta_{j}+\pi\right) F_{12}(\gamma-\mathrm{i} \theta) \bar{F}_{12}\left(\gamma-\mathrm{i} \theta_{j}+\mu+\pi\right) \\
& \times \prod_{j \in N_{-}} \bar{F}_{12}\left(\gamma-\mathrm{i} \theta_{j}-\mathrm{i} \nu_{j}+\pi\right) \bar{F}_{12}\left(\gamma-\mathrm{i} \theta_{j}-\mathrm{i} \nu_{j}\right) \overline{\bar{F}}_{12}\left(\gamma-\mathrm{i} \theta_{j}+\mu-\mathrm{i} \nu_{j}+\pi\right), \\
S_{+-}(\gamma, \mu ; \varepsilon, \theta, \nu)= & \prod_{j} F_{12}\left(\gamma-\mathrm{i} \theta_{j}+\pi\right) F_{12}(\gamma-\mathrm{i} \theta) \bar{F}_{12}\left(\gamma-\mathrm{i} \theta_{j}+\mu\right) \\
& \times \prod_{j \in N_{-}} \bar{F}_{12}\left(\gamma-\mathrm{i} \theta_{j}-\mathrm{i} \nu_{j}+\pi\right) \bar{F}_{12}\left(\gamma-\mathrm{i} \theta_{j}-\mathrm{i} \nu_{j}\right) \overline{\bar{F}}_{12}\left(\gamma-\mathrm{i} \theta_{j}+\mu-\mathrm{i} \nu_{j}\right),
\end{aligned}
$$

and it is equal to 1 up to $O(\epsilon)$. But the ratio can be estimated much more precisely by use of Eq. (4.3):

$$
\begin{aligned}
\frac{S_{+-}(\gamma, \mu ; \varepsilon, \theta, \nu)}{S_{-+}(\gamma, \mu ; \varepsilon, \theta, \nu)} & =\prod_{j} \frac{\bar{F}_{12}\left(\gamma-\mathrm{i} \theta_{j}+\mu\right)}{\bar{F}_{12}\left(\gamma-\mathrm{i} \theta_{j}+\mu+\pi\right)} \prod_{j \in N_{-}} \frac{\overline{\bar{F}}_{12}\left(\gamma-\mathrm{i} \theta_{j}+\mu-\mathrm{i} \nu_{j}\right)}{\overline{\bar{F}}_{12}\left(\gamma-\mathrm{i} \theta_{j}+\mu-\mathrm{i} \nu_{j}+\pi\right)}=\mathrm{e}^{\mathrm{i} \epsilon \Theta / \pi}+O(q), \\
\Theta & =\sum_{j \in N_{+}} \theta_{j}-\sum_{j \in N_{-}}\left(\theta_{j}+2 \nu_{j}\right) .
\end{aligned}
$$

Note that $\Theta=\mathrm{i} \pi B^{\prime} / \sqrt{2}$ with $B^{\prime}$ being the contribution of $\tilde{V}_{\varepsilon}$ into $B$ from Eq. (3.7).

We conclude that the factor $F_{-\alpha \alpha}(\gamma, \mu ; \varepsilon, \theta, \nu)$ is independent of $\alpha$ up to $O(\epsilon)$, but we are able to calculate the ratio $F_{+-} / F_{-+}$with much better precision, namely up to $O(q)$. This precision allows us to treat the terms with $n= \pm 1$ in Eq. (3.9), which are of the order $q^{1 / 2}$.

Now consider the zero mode contribution $H_{-\alpha \alpha}$. From Eq. (3.8) we obtain

$$
\begin{aligned}
H_{-\alpha \alpha}^{(i)}(\gamma, \mu ; \varepsilon, \theta, \nu)= & \mathrm{e}^{-\mathrm{i} \epsilon A^{\prime}-\frac{\epsilon}{2}\left(\frac{2 \gamma}{\pi}-1\right)} \mathrm{e}^{-\frac{2 \epsilon \mu}{\pi} \frac{\alpha+1}{2}} \exp \left[4 \epsilon\left(\frac{1-\alpha}{4}+\frac{B^{\prime}}{4 \sqrt{2}}-\frac{\mu}{2 \pi}\right)^{2}-\frac{\epsilon}{4}\right] \\
& \times \theta_{3+i}\left(\frac{1-\alpha}{4}+\frac{B^{\prime}}{4 \sqrt{2}}-\frac{\mu}{2 \pi} ; \frac{\mathrm{i} \pi}{4 \epsilon}\right) / \theta_{3+i}\left(\frac{1}{4} ; \frac{\mathrm{i} \pi}{4 \epsilon}\right),
\end{aligned}
$$

where $A^{\prime}$ and $B^{\prime}$ are contributions of $\tilde{V}_{\varepsilon}$ into the constants $A$ and $B$ from Eq. (3.7). Collecting equations (4.8), (4.12), and (4.13) we obtain for the functions in the integrand of Eq. (4.6)

$$
\frac{H_{+-} F_{+-}}{H_{-+} F_{-+}}=\theta_{4-i}\left(\frac{B^{\prime}}{4 \sqrt{2}}-\frac{\mu}{2 \pi} ; \frac{\mathrm{i} \pi}{4 \epsilon}\right) / \theta_{3+i}\left(\frac{B^{\prime}}{4 \sqrt{2}}-\frac{\mu}{2 \pi} ; \frac{\mathrm{i} \pi}{4 \epsilon}\right)+O(q)
$$

[recall that $\left.\theta_{4}(u)=\theta_{3}( \pm 1 / 2+u)\right]$. It means that the difference $F_{-+}-F_{+-}$is proportional to the difference of theta functions in the scaling limit,

$$
\theta_{3+i}(u ; \mathrm{i} \pi / 4 \epsilon)-\theta_{4-i}(u ; \mathrm{i} \pi / 4 \epsilon)=(-)^{i} q^{1 / 2} \cdot 4 \cos 2 \pi u+O(q) .
$$

In our case

$$
u=-\frac{\mu}{2 \pi}+\frac{B^{\prime}}{4 \sqrt{2}}=-\frac{1}{2 \pi}\left(\mu-\frac{\mathrm{i}}{2} \Theta\right) .
$$

We see that the contributions of the order $q^{0}$ [terms with $n=0$ in Eq. (3.9)] cancel each other in $\sigma^{z}$, and all form factors are comparable and proportional to $q^{1 / 2} \sim M^{1 / 2}$. Once we extracted the factor of this order, we may only take into account the terms of the order $\epsilon^{0}$ in all other multipliers. We obtain the expression

$$
\begin{aligned}
& \left\langle 0\left|\sigma^{z}(0)\right| \varepsilon_{1} \theta_{1}, \ldots, \varepsilon_{2 n} \theta_{2 n}\right\rangle_{\mathrm{in}}^{(i)} \\
& \quad=(-)^{1-i} 2(b M)^{1 / 2} \int_{C_{1}^{\prime}} \frac{d \mu}{2 \pi \mathrm{i}} \int_{C_{2}^{\prime}} \frac{d^{n} \nu}{(2 \pi)^{n}} F_{-+}(\mu) F(\varepsilon, \theta, \nu) \cos \left(\mu-\frac{\mathrm{i}}{2} \Theta\right),
\end{aligned}
$$


where $F_{-+}(\mu)$ and $F(\varepsilon, \theta, \nu)$ are given by approximate equations (4.9) and (4.10). We see that the integrand nearly splits into two factors, one of which only depends on $\mu$ and the other one on $\nu_{j}$. The only factor depending on both $\mu$ and $\nu_{j}$ is the 'zero mode' factor $\cos \left(\mu-\frac{i}{2} \Theta\right)$. Note that the function $F_{-+}(\mu)$ is even. Therefore, we may substitute $\cos \left(\mu-\frac{i}{2} \Theta\right)$ in the integrand by $\cos \mu \cos \frac{i}{2} \Theta$, and the integral factorizes completely. Consider the function $F_{-+}(\mu) \cos \mu$. Calculating it numerically by use of the Mathematica package I found it to be a constant:

$$
F_{-+}(\mu) \cos \mu=-1 .
$$

Hence the integral in $\mu$ is linearly divergent. This is the problem mentioned above. Therefore we should consider large $|\mu| \sim \epsilon^{-1}$. Note, that Eqs. (4.1c), (4.3), (4.8), (4.12), and therefore (4.14) are valid in the case of large $\mu$. The equation (4.15) is valid in the whole region $-\pi^{2} / 2 \epsilon<\operatorname{Im} \mu<\pi^{2} / 2 \epsilon$ except small vicinities of the ends. To find approximations of $F_{11}, C_{-}$in the region $|\mu| \gg 1$ one can expand summands in $\epsilon n$ everywhere except the places where $\epsilon n$ enters with a large coefficient $\mu$, then one can sum the series in the regions of convergence and continue analytically the answers. Such consideration shows that the integrands in all form factors of $\sigma^{z}$ remain factorable and constant in $\mu$ in nearly the whole region $-\pi^{2} / 2 \epsilon<\operatorname{Im} \mu<\pi^{2} / 2 \epsilon$. On the other hand the integration region in $\mu$ for finite $\epsilon$ must be precisely $\left[-\mathrm{i} \pi^{2} / 2 \epsilon, \mathrm{i} \pi^{2} / 2 \epsilon\right]$.

So we finally obtain

$$
\begin{aligned}
\left\langle 0\left|\sigma^{z}(0)\right| \varepsilon_{1} \theta_{1}, \ldots, \varepsilon_{2 n} \theta_{2 n}\right\rangle_{\mathrm{in}}^{(i)} & (-)^{i} \frac{2}{\pi}(b M)^{1 / 2} \log \left(\frac{4}{b M}\right) \int_{C_{2}^{\prime}} \frac{d^{n} \nu}{(2 \pi)^{n}} F(\varepsilon, \theta, \nu) \operatorname{ch} \frac{\Theta}{2} \\
= & (-)^{i}\left(\frac{2}{\pi}\right)^{n+1}(b M)^{1 / 2} \log \left(\frac{4}{b M}\right) \tilde{c}_{+}^{n} \tilde{c}_{-}^{n} \int_{C_{2}^{\prime}} \frac{d^{n} \nu}{(2 \pi)^{n}} \operatorname{ch}\left(\frac{1}{2} \sum_{j \in N_{+}} \theta_{j}-\frac{1}{2} \sum_{j \in N_{-}}\left(\theta_{j}+2 \nu_{j}\right)\right) \\
& \times \prod_{j \in N_{-}} f_{2}(\nu) \prod_{j<j^{\prime}} f_{22}\left(\theta_{j^{\prime}}-\theta_{j}\right) \prod_{j<j^{\prime}, j^{\prime} \in N_{-}} \bar{f}_{22}\left(\theta_{j^{\prime}}-\theta_{j}+\nu_{j^{\prime}}\right) \prod_{j<j^{\prime}, j \in N_{-}} \bar{f}_{22}\left(\theta_{j^{\prime}}-\theta_{j}-\nu_{j}\right) \\
& \times \prod_{j<j^{\prime} \in N_{-}} \overline{\bar{f}}_{22}\left(\theta_{j^{\prime}}-\theta_{j}+\nu_{j^{\prime}}-\nu_{j}\right) .
\end{aligned}
$$

For $n=0$ it matches the well known Baxter-Kelland formula ${ }^{13}$

$$
(-)^{i}\left\langle\sigma^{z}\right\rangle^{(i)}=\frac{\left(x^{2} ; x^{2}\right)_{\infty}^{2}}{\left(-x^{2} ; x^{2}\right)_{\infty}^{2}} \simeq \epsilon^{-1} q^{1 / 2}(2 \pi+O(q))
$$

Let us interpret the result from the point of view of quantum field theory. First of all, note that every form factor is proportional to $M^{1 / 2}$. It means that the scaling dimension of the field $\sigma^{z}(x)$ is $1 / 2$. There are two linearly independent local fields of this dimension in the critical sine-Gordon theory, $\mathrm{e}^{\mathrm{i} \varphi / 2}$ and $\mathrm{e}^{-\mathrm{i} \varphi / 2}$. We expect that the field $\sigma^{z}(x)$ is a linear combination of these fields. Comparison with the result of Ref. 9 supports this conclusion and makes it possible to calculate coefficients of the combination. The decomposition of $\sigma^{z}$ corresponds to the decomposition of $\operatorname{ch} \frac{1}{2} \Theta$ into two exponentials in Eq. (4.17). To make it clear, let us pass from the bosonization of the lattice model to the bosonization of the field theory.

The 'conjugate zero mode' operators $P^{\prime}$ and $Q^{\prime}$ survive in the scaling limit, but the coefficient at $P^{\prime 2}$ in $D^{\prime(i)}$ tends to infinity. So we may only take into account $P^{\prime}$. The only trace of $Q^{\prime}$ lies in the requirement $\sum_{j} \alpha_{j}=0$. Considering form factors of lattice objects like $\sigma^{z}$ we must accurately extract the leading terms in (3.9), as we have done above. But for the field theory operators we can consider any expectation $\left\langle P^{\prime}|\ldots| P^{\prime}\right\rangle$. For $\epsilon \rightarrow 0$ it takes the form

$$
H_{N}^{(i)}\left(P^{\prime}\right)=(b M / 4)^{4 P^{\prime 2} / \pi} \mathrm{e}^{\mathrm{i}(-1)^{i} \sqrt{2} P^{\prime}} \mathrm{e}^{\mathrm{i} \beta_{N} P^{\prime}} \ldots \mathrm{e}^{\mathrm{i} \beta_{1} P^{\prime}} .
$$

It means that the zero mode contribution to the scaling dimension of the fields is equal to $4 P^{\prime 2} / \pi^{2}$. The contribution (4.18) just provides the factors before exponent field in Lukyanov's formula for vertex operators corresponding to the field $\mathrm{e}^{\mathrm{i} a \varphi}$ for $P^{\prime}=\pi a / \sqrt{2}$. Note that in this case $4 P^{\prime 2} / \pi^{2}=2 a^{2}$ gives the correct conformal dimension of the field $\mathrm{e}^{\mathrm{i} a \varphi}$. 
Consider the oscillators $a_{n}$. It is tempting to think that the set of $a_{n}$ tends in the scaling limit to a continuous set of operators $a(\eta)$. But it is not quite correct. To see it, let us consider the field $F_{12}(\gamma)$. The expression for this correlation function contains the factor $(-1)^{n}$ [see Eq. (3.12)], which is rapidly oscillating in the scaling limit for finite $\gamma$. It reflects the fact that the oscillators forming $V_{ \pm}$differ from those forming $\tilde{V}_{ \pm}$by the factor $(-1)^{n}$ [see Eqs. (3.1), (3.3)]. It means that the set of $a_{n}$ splits in two continuous sets of operators $a(\eta)$ and $b(\eta)$,

$$
\left[a(\eta), a\left(\eta^{\prime}\right)\right]=\left[b(\eta), b\left(\eta^{\prime}\right)\right]=\delta\left(\eta+\eta^{\prime}\right) \frac{\operatorname{sh} 2 \eta \operatorname{sh} \eta}{\eta}, \quad\left[a(\eta), b\left(\eta^{\prime}\right)\right]=0 .
$$

Now let us define the operators $\phi(\gamma)$ and $\tilde{\phi}(\theta)$ as follows ${ }^{c}$

$$
\begin{aligned}
& \phi(\gamma)=\frac{\sqrt{2}}{\pi} P^{\prime} \gamma-\int_{-\infty}^{\infty} d \eta \frac{a(\eta)}{\mathrm{i} \operatorname{sh} 2 \eta} \mathrm{e}^{-|\eta| / 2} \mathrm{e}^{\frac{2}{\pi} \eta \gamma} \\
& \tilde{\phi}(\theta)=-\frac{\mathrm{i} \sqrt{2}}{\pi} P^{\prime} \theta+\int_{-\infty}^{\infty} d \eta \frac{b(\eta)}{\mathrm{i} \operatorname{sh} 2 \eta} \mathrm{e}^{|\eta| / 2} \mathrm{e}^{\mathrm{i} \frac{2}{\pi} \eta \theta} .
\end{aligned}
$$

Finally the definition of the vertex operators is the following

$$
\begin{aligned}
& V_{+}(\gamma)=g^{1 / 2}: \mathrm{e}^{\mathrm{i} \phi(\gamma)}: \\
& V_{-}(\gamma)=g^{1 / 2} \int_{C_{1}^{\prime}} \frac{d \mu}{2 \pi \mathrm{i}} \frac{\pi}{\mu^{2}-\pi^{2} / 4}: \mathrm{e}^{\mathrm{i} \phi(\gamma)-\mathrm{i} \phi(\gamma+\mu-\pi / 2)-\mathrm{i} \phi(\gamma+\mu+\pi / 2)}: \\
& \tilde{V}_{+}(\theta)=\sqrt{\frac{2}{\pi}} g^{-1 / 2}: \mathrm{e}^{\mathrm{i} \tilde{\phi}(\theta)}: \\
& \tilde{V}_{-}(\theta)=-\sqrt{\frac{2}{\pi}} g^{-1 / 2} \int_{C_{2}^{\prime}} \frac{d \nu}{2 \pi} \frac{\pi}{\nu^{2}+\pi^{2} / 4}: \mathrm{e}^{\mathrm{i} \tilde{\phi}(\theta)-\mathrm{i} \tilde{\phi}(\theta+\nu+\mathrm{i} \pi / 2)-\mathrm{i} \tilde{\phi}(\theta+\nu-\mathrm{i} \pi / 2)}:
\end{aligned}
$$

with

$$
\log g=\int_{0}^{\infty} \frac{d \eta}{\eta} \frac{\mathrm{e}^{-\eta}(\operatorname{ch} \eta-1)}{2 \operatorname{ch} \eta}
$$

if we assume the regularization rule

$$
\int_{0}^{\infty} d \eta\left(\frac{a_{2}}{\eta^{2}}+\frac{a_{1} \mathrm{e}^{-\eta}}{\eta}+f(\eta)\right) \longrightarrow \int_{0}^{\infty} d \eta f(\eta), \quad \int_{C_{1}^{\prime}} \frac{d \mu}{2 \pi \mathrm{i}} \text { const. }=\frac{\pi}{2 \epsilon} \text { const. }
$$

for $f(\eta)$ finite at $\eta \rightarrow 0$.

The vertex operators $\tilde{V}_{ \pm}(\theta)$ are Lukyanov's vertex operators from Ref. 9. The factors before the exponential operators are hidden in the zero mode $P^{\prime}$. The operators $V_{ \pm}(\gamma)$ can be considered as the primed operators from Ref. 3 at the infinitely distant point. They only interact with the operators $\tilde{V}_{ \pm}(\theta)$ through the zero mode. Their physical meaning in the framework of the quantum field theory is vague. As we can see, the initial idea by Lukyanov that the primed vertex operators describe local fields was in the right direction, but it is necessary to move them to the point $\pi^{2} / 2 \epsilon$ which is infinitely far for $\epsilon \rightarrow 0$.

Comparison with Ref. 9 gives

$$
\sigma^{z}(x) \sim \cos \frac{1}{2} \varphi(x)
$$

Unfortunately we are unable to find the proportionality coefficient from the vacuum expectations, found by Lukyanov in Ref. 11. In the limit $\beta \rightarrow 1$ these expectation values become infinite. It means that the critical sine-Gordon is not described by a free boson in the ultraviolet region, and the normalization of the operators of Ref. 11, based on the conformal field theory, is not applicable in this case. In the lattice theory it is reflected by the additional factor $\epsilon^{-1} \sim \log (4 / b M)$ in the form factors. This factor means that

$c$ The terms $\sim \log x^{3}$ in (3.3) do not contribute traces in this limit. In the exact formulas they can be also omitted together with the factors $x^{3 / 4}$ in $(3.1)$. 
the normalization changes with the scale. Maybe it witnesses about logarithmic factors in the correlation functions at short distances.

Our last remark concerns the fields ${ }^{6,14} \sigma^{ \pm}=\frac{1}{2}\left(\sigma^{x} \pm \mathrm{i} \sigma^{y}\right)$. The leading zero mode contribution to these fields comes from $n=0$ in (3.9), but the scaling dimension $1 / 2$ comes from the oscillators, because the requirement $\sum_{j} \alpha_{j}=0$ gives here $\# N_{+}-\# N_{-}= \pm 2$, and the factors depending on $q$ in (4.1) and (4.4) do not cancel. It is easy to understand from the form factors axioms ${ }^{3}$ that the fields $\sigma^{ \pm}(x)$ are semilocal with respect to $\sigma^{z}(x)$. There are two semilocal fields in the critical sine-Gordon theory $\mathrm{e}^{ \pm \mathrm{i} \tilde{\varphi} / 2}$ with $\tilde{\varphi}(x)=\int_{-\infty}^{x^{1}} d y^{1} \partial_{0} \varphi(y)$. So we can suppose that

$$
\sigma^{ \pm}(x) \sim \mathrm{e}^{ \pm \frac{\mathrm{i}}{2} \tilde{\varphi}(x)}
$$

\section{Discussion}

We conclude that the explicit form of the form factors in the scaling limit of the six vertex model is consistent with Lukyanov's conjecture on the free field representation of the form factors of the exponential fields in the sine-Gordon model for $\beta=1, a=1 / 2$. A plausible argumentation extends this consistency to arbitrary values of $a$. This investigation can be continued in two ways. First, it would be interesting to calculate form factors of more general local fields, e. $g$. products of adjacent polarizations. Second, it is important to generalize this derivation to other models. For example, the bosonization of the Andrews-Baxter-Forrester model $^{12}$ must give in the scaling limit the bosonization for the restricted sine-Gordon model.

We expect that the divergency in the integral in $\mu$ is a peculiarity of the six vertex model or of the critical sine-Gordon model. It is related to the logarithmic factors in the scaling behavior of quantities, and originates in the dynamical mass generation phenomenon.

\section{Acknowledgments}

I am grateful to R. Schrader for his hospitality at Freie Universität Berlin where this work was started under the support by the program Volkswagen-Stiftung. I am indebted to B. Davies, B. Feigin, S. Khoroshkin, D. Lebedev, S. Lukyanov, S. Pakuliak, Ya. Pugai, and J. R. Reyes Martínez for fruitful discussions, and, especially, to P. Grinevich for his consultations on elliptic functions. This work was supported by Civilian Research and Development Foundation under the grant RP1-277, and by INTAS and RFBR under the grant 95-0690.

\section{References}

1. A. B. Zamolodchikov and Al. B. Zamolodchikov, Annals in Phys. 120, 253 (1979)

2. F. A. Smirnov, Form Factors in Completely Integrable Models of Quantum Field Theory, World Scientific, Singapore, 1992

3. S. Lukyanov, Commun. Math. Phys. 167, 183 (1995) hep-th/9307196

4. B. Davies, O. Foda, M. Jimbo, T. Miwa, and A. Nakayashiki, Commun. Math. Phys. 151, 89 (1993)

5. M. Jimbo and K. Miki, T. Miwa, and A. Nakayashiki, Phys. Lett. A168 256 (1992)

6. M. Jimbo, T. Miwa, Algebraic Analysis of Solvable Lattice Models, CBMS Regional Conference Series in Mathematics, vol. 85, AMS, 1994

7. R. J. Baxter, Exactly Solved Models in Statistical Mechanics, Academic Press, 1982

8. A. Luther, Phys. Rev. B14, 2153 (1976)

9. S. Lukyanov, Form-factors of exponential fields in the sine-Gordon model, CLNS 97/1471, March 1997, hep-th/9703190

10. M. Jimbo, H. Konno, and T. Miwa, Massless $X X Z$ model and degeneration of the elliptic algebra, October 1996, hep-th/9610079

11. S. Lukyanov and A. Zamolodchikov, Exact expectation values of local fields in quantum sine-Gordon model, CLNS 96/1444, RU-96-107, hep-th/9611238 
12. S. Lukyanov and Ya. Pugai, Multi-point local height probabilities in the integrable RSOS model, February 1996, hep-th/9602074

13. R. J. Baxter and S. B. Kelland, it J. Phys. C7, L403 (1974)

14. A. Abada, A. H. Bougourzi, and B. Si-Lakhal, Exact four-spinon dynamical correlation function of the Heisenberg model, ITP-SB-96-72, December 1996, hep-th/9702028 